\title{
Improvement of Confusion Matrix for Hand Vein Recognition Based On Deep- Learning multi-classifier Decisions
}

\author{
Nadia. M. Nawwar ${ }^{1}$ ， Hani. Kasban ${ }^{2}$ and May .salama ${ }^{3}$ \\ ${ }^{(1)}$ Department of Nuclear Fuel Technology, Hot labs Center, Egyptian Atomic Energy Authority, Cairo, Egypt. \\ (2) Engineering Department, Nuclear Research Center, Egyptian Atomic Energy Authority, Cairo, Egypt. \\ ${ }^{(3)}$ Electrical Engineering Department, Faculty of Engineering at Shoubra, Banha University, Egypt
}

\begin{tabular}{l} 
ARTICLE INFO \\
Article history: \\
Received: $1^{\text {st }}$ Apr. 2021 \\
Accepted: $8^{\text {th }}$ July 2021 \\
\hline
\end{tabular}

Keywords:

Biometric,

Hand vein,

CNN, GAN

FAR, FRR,

Confusion matrix.

\section{ABSTRACT}

In this paper, a recognition of the hand vein patterns approach is proposed employing the Convolutional Neural Network (CNN). This approach is routinely well-learned in what way to get features from the main pattern using Region of Interest (ROI). Though, the poor quality of the hand vein image still attitudes an unlimited strain to the extension leads of its usability. Firstly, by applying the method of Generative adversarial networks (GAN) data augmentation, the performance gain of adding GAN generatdata exceeds that of adding more true images, and apply ROI in a hand vein image feature extraction which is studied initially. Secondly, the suggested approach is tested on the data sets of hand veins to decrease the overfitting in the fully connecting layer of $\mathrm{CNN}$ which this model proves the most effective one. In total, 1575 hand vein images from 100 subjects are applied to authorize the proposed approach for hand vein. A high accuracy $(>99.8 \%)$ and low False Rejection Rate(FRR) $(<0.99 \%)$ were achieved by applying the suggested approach, when compared with the existing CNN classifiers, indicating the efficiency of the suggested approach.

\section{INTRODUCTION}

In recent years, Biometrics utilizes metrics related to the human biological and behavioral features, the biometrics acts as an imperious character in-person verification [1]. A new environmental science feature recognition machinery - hand vein has been planned in this research. Matching all the biometric individualities, the hand vein modalities have several benefits, as the vein in hand is the internal feature of the body, and it cannot be faked, which veins are lifeblood carrying containers interweaved with physiques and bones so the vein recognition is contactless, hand vien do not contact with the body of human .Finally, the vein are long-term features [2-4]. Thus, the recognition machinery established on vein patterns features in order to develop a current emergent tendency of biometrics.

The hand vein recognition approach is collected by the image gaining [5-7], the image enhancement, the ROI of feature drawing out, identical image, and the result fusion [8, 9]. It is usually accepted before feature drawing out to reduce the noise influence on the consequent feature abstraction and to increase enactment. Toward adapt the main patterns into the necessary designs, the pre-processing operation will be approved with on the original patterns [10-12]. Depending on [13-16] deep learning techniques have been supported in image classification and responsibilities recovery. The usage vein recognition approach from side to side Deep learning DL-based and CNN architecture applications.

According to other researchers [14, 17, 18], planning a forceful approach for recognition of a vein is a routine. The main challenge originates from the lack of a quick and resourceful way to extract matchless features. Based on the minutiae points of vein images as mentioned in previous studies [6, 19-21], we should pull out endpoint features and the borderline distances between the two endpoints alongside the margins of every image. [22-24]. The original approach of feature drawing out proposals 
the density of features vein patterns and demonstrates the vein characteristic suitably [25-27].

The rest of the sections of the paper are arranged as follows. Section 2 briefly presents related works. A detailed description of the proposed approach is given in sections 3. Experimental results are presented in sections 4.Finally, conclusion is highlighted in section 5

\section{RELATED WORKS}

The important characteristic of the hand vein approach is vein recognition built on gray features and vein recognition based on points and arcs as previously reported [2, 28], and also categorized into 3 categories: appearance, multiple fusion, and geometry [29,.30, 31]. In this section, some of the vein recognition systems will be presented.

A previous study [7] recommended a method for a multimodal biometric approach using vein patterns of the hand such as a vein of the finger and hand palm, and the technique runs lower FAR, FRR, and it is of a accuracy. Yuhang et al. [32] introduced a comparatively good recognition effect, which the technique of extracting a hand vein pattern constructed on end and crossing points. Bashar et al. [10] achieved proportional learning to identify feature-classifier mixture that creates well-organized vein biometric approaches. 7 texture and three multiclass classifiers were discovered near the managed ID of individuals from a vein.Lee et al. [16] introduced feature mining outclasses the broadly used Gabor filters, which was appropriately wild for actual verification, and by comparing this, the proposed approach can result in an enhanced performance. Kumar et al. [33] demonstrated a new method to confirm persons using the concurrent abstraction of knuckle shape figures. Kumar et al., in another study [34] introduced hand vein patterns by a convolution neural network, the evaluation of consequences suggested prototypical model is complete with other $\mathrm{CNN}$ models like VGG-19. The marks of the experiments display that the suggested prototype (finetune of VGG16) attains advanced accuracy of recognition. $\mathrm{Li}$ et al. [21] proposed a DPT to fast-track identical performance and assessed the prejudiced power of the feature area for confirming one's individuality. The suggested technique got the maximum accuracy in the lowermost tested time. Pflug et al. [35] suggested a novel restraint code that founded encoding technique, using longitudinal and positioning belongings of the vein, where is skilled in distributing with deafening and unbalanced skeletons. Chain code assessment and a choice of pre-processing procedures were used to assess in a sequence of diverse trials in single and multi-reference situations on two dissimilar vein data sets. The trials displayed that chain code assessment outclasses details methodologies and comparison constructed mixture identical

Huang et al.[36] recommends a new methodology to hand vein model over matching features of multiple bases. In a comparison of present pieces of training only thinking on the hand vein system, the suggested approach assumes an actual key point recognition approach to focus features on hand vein. As individual authentication has developed a significant and great request technique for security admission approaches in previous period.[37], the contour of the interior vine hierarchy of the backbone of the hand comprises figures that are accomplished of verifying the individualism of an individual to a sensible accuracy for programmed personal authentication resolves. In earlier publications [38-40], the authors had an acceptable result signifying that in vein biometrics whichever confirmation or ID determinations, nevertheless of the dissimilarity in data set size, techniques, or vein likenesses used. ROI and background removal, flattening and noise decline, native thresholding for splitting veins, and post-processing were previously reported $[39,41,42]$.

Lajevardi et al. [43] suggested a programmed hand vein confirmation method using a innovative algorithm called biometric graph identical by using minor and short templates.

Sontakki et al.[44] proposed a prototypical is used to advance the accuracy and reply time of vein verification and use NNs for the last estimation to identify the individual. Raghvanedra et al.[45] presented a new sensor detects a good feature of vein images. The existing sensor services a particular camera with a particular construction that will additionally recover the excellence of the light to right brighten the vein region also proposed a widespread assessment of 8 varities state-of-the-art methods that confirmed the remaining enactment of the Log-Gabor and SRCwith an error equal rate of $0.7 \%$. In a previous study [46] ,the author introduced different preprocessing techniques. Through experimentation, the outcome shows that extracted vein image bounces improved enactment and pointers to a more protected biometric verification approach. Qin et al.[47] introduced deep learning prototypical to remove and improve vein structures using limited priory figures. A $\mathrm{CNN}$ is accomplished on the resultant data set to calculate the possibility of each vein 
interesting point and has achieved better performance too robustly.

Kang et al. [48] suggested contactless vein established on communal local binary design. Even if it also used change matching, its process is for the contactless vein. VGG 16 planned with new and dissimilarity patterns as input were tuned and structures were taken out from the layer before the last fullyconnected layer. Zhou et al. [49] concluded that most of the trained system are in use. The system which routines variance patterns as the input creates two outputs from a single input.

Other studies [50, 51] introduced a feature-ranking algorithm and the outcomes were assessed for the kernelNN classifier prototypical formed sideways with the suggested procedure, an ID of $90.44 \%$ for the 1 st structure, $94.44 \%$ for the 2 nd structure, and $87.22 \%$ for the 3 rd structure has effectively been achieved. Sohrabi et al.[52] introduced a structure for consumers' interactive sketching construction is accessible and client interactive designs are used for client ID in the e-Commerce surroundings. The NNs arrangement and the amount of comparison between social arrangements are two attitudes realistic in this proposal. The outcomes of procedure designate are less error and up to $15.12 \%$. The outcomes recommend that the precision of the NNs attitude in client design manners recognition growths when the number of clients grows.

\section{III.Proposed approach}

\section{A. CNN in use}

Convolutional Neural Network is one of the greatest consequences and widely used constructions in the deep learning community generally for computer vision responsibilities which is a class of deep NNs. Lecun et al. [53] builtthe architecture of one of the first $\mathrm{CNN}$ models. Fig. (1) illustrates CNN's mostly consists of three type of layers: convolutional layers, where a sliding kernel is practical to the image in command to extract features; nonlinear layers, which spread on an activation function on the features in command to allow the demonstrating of non-linear tasks by the system; and pooling layers, which takes a small area of the feature map and substitutes it employing some arithmetical evidence of the area. There are multi-constructions of the CNN model. The most common models are VGG16 (explained in Table (2) andVGG19 (explained in Table (3).

The soft-max function is used to categorize the purpose's possibility where the function returns 1 if the purpose is acknowledged if not 0 , which is used lastly to choose which one of the matched pattern is authorized or unauthorized. Nodes in the CNN layers are in the vicinity linked; that is to say, each unit with a layer gets input from a minority neighborhood of the receptive field. In the proposed approach as shown in the construction in Table (4) the different filter of size $3 * 3$ for the first and second layers and $5 * 5$ for third and fourth layers with stride(2) then an only max-pooling layer applied afterward uses 3 fully linked layer with Relu and softmax layer with a single dropout layer 04 .

The foremost advantage of $\mathrm{CNN}$ is the weightiness sharing mechanism through the usage of the sliding kernel, which goes over the images, and aggregates the local information to extract the features. $\mathrm{CNN}$ has a significantly smaller number of parameters compared to a fully connected neural network. Additionally, by stacking many convolution layers, the upper-level layers learn features from progressively ROI.

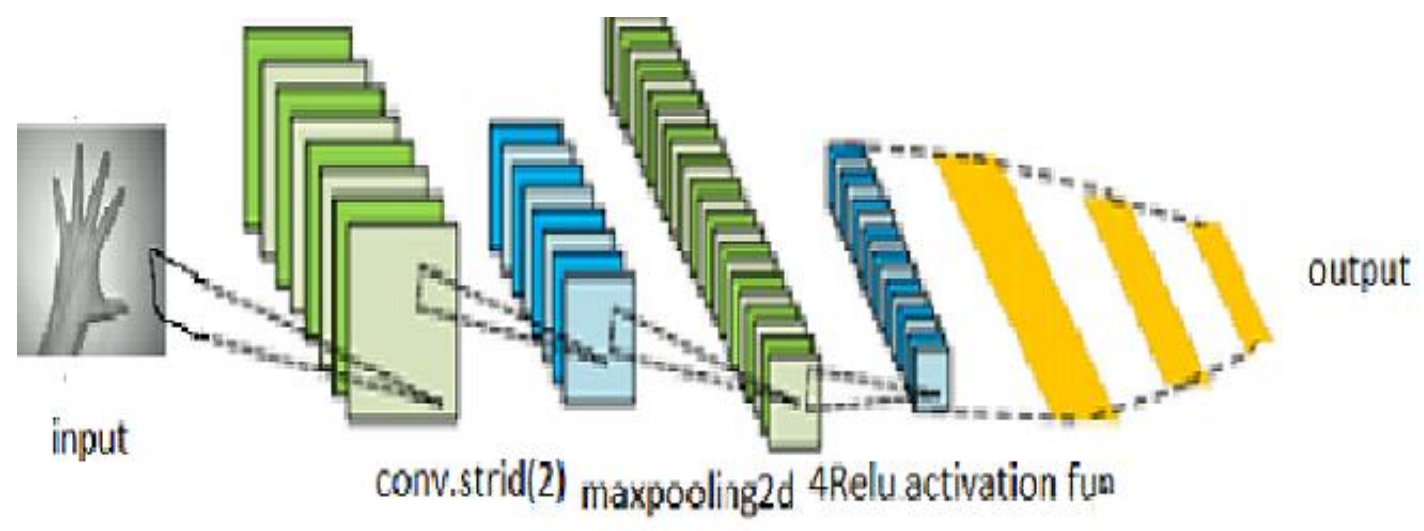

Fig. (1): CNN in use 


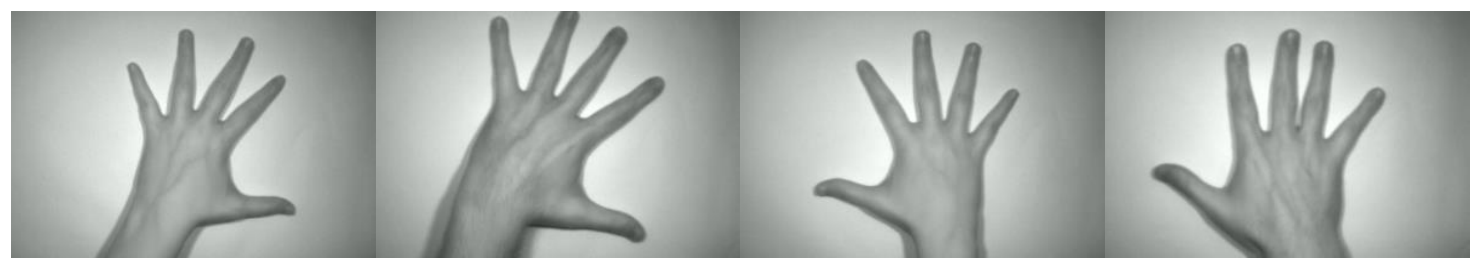

Fig. (2): Samples multispectral hand vein database in left and right hand

\section{B. Hand vein Data set}

This paper used a large dataset associated with the hand vein pattern which is recognized as the Bosphorus vein pattern which is developed using irradiated two IR light sources. The NIR CCD camera is well-found with an IR lens. All patterns have $300 \times 240$ pixel size with the grayscale perseverance of 8-bits. The total images are 1575 for 100 subjects circulated as follows:

- In normal case 3 left-hand images per object,

- In case of carrying a bag weighing $3 \mathrm{~kg} 3$ lefthand images per object,

- In case of closing and opening an elastic ball 3 left-hand images per object,

- In the case of holding an ice pack 3 left-hand images per object,

- $\quad$ Finally, In a normal case 3 right-hand images per object.

\section{GAN data augmentation}

Generative adversarial networks are powerful Dl-based generative approach that has new patterns, and the discriminator approach that attempts to classify patterns as either real data set. In this paper, training GANs on the patterns which have the most effective facility to perfect high-dimensional data sets was proposed that can lead to the dramatic improvement in classifier performance. For all modules, a dropout rate of 0.1 after the $1 \mathrm{st}, 2 \mathrm{nd}$, and 3rd hidden layers was used after many trials to avoid overfitting. To get a better vein pattern learning time was reduced by reducing epoch number to 50, while the training is in progress the generative approach learns to generate the actual vein patterns after 30 epoch.

\section{The proposed algorithm}

The hand vein construction can not be simply seen. The brightness of the vein construction differs considerably according to features such as age, levels of hypodermal overweight, Ambien heat and moistness, and bodily activity. Adding an assembly of other features, including the exterior layer can opaque the image. In the present study, datasets, images were changed into a binary image. The proposed approach diagram for the hand vein pattern module is established in Fig. (3)

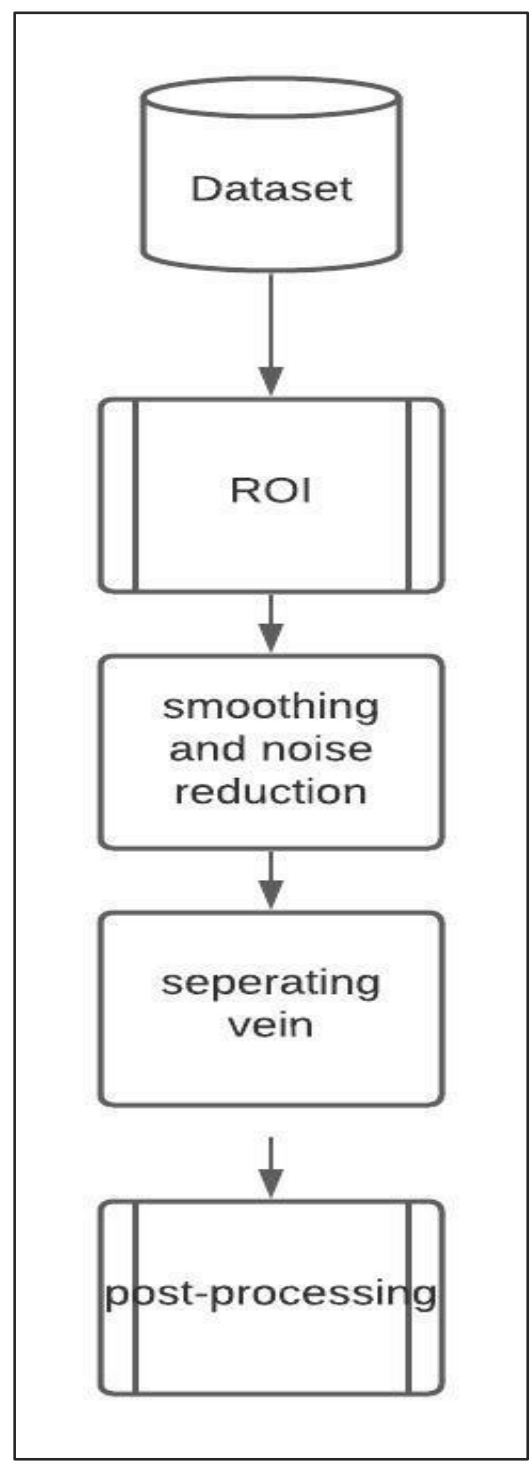

Fig. (3): Block diagram of the processing stage 


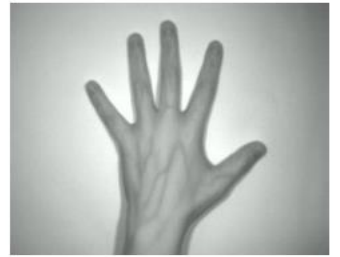

(a)

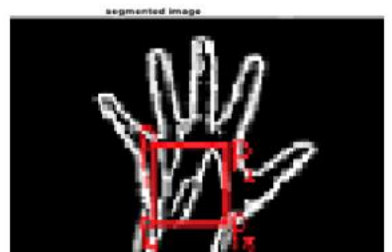

(b)

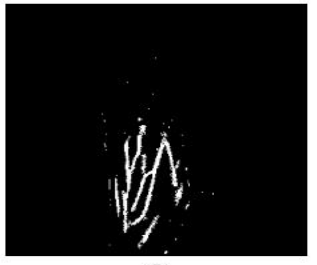

(C)

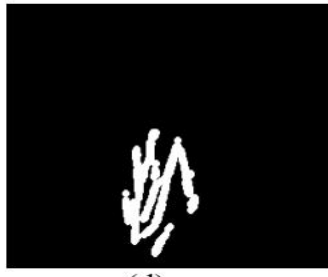

(d)

Fig. (4): vein recognition process (a) original image, (b-d) apply average filter $9 * 9$ to get adaptive threshold

The objective of pre-prossing is to extract features from the vein patterns. To advance accuracy, the nonvein interesting areas are identified by contrast enhancement of gray image by using adapt histogram equalization then, applying average filter $9 * 9$ to revenue the dissimilarity among gray image and average filter after that gets adaptive threshold to convert image to a binary one and to remove pixels less than 100 pixels. Finally, boundary and vein of hand are converted into white color, which is applied by a laptop setting (Intel(R) Core (MT) i7-7700 HQ CPU @ 2.80 GHz,win10 64-bit operating approach, RAM16 GB, 256GB SSD, NVIDIA GTX 1060 graphics card and applied by Matlab 2018b. Fig. (4) shows the segmenting of pattern into two points; a body (hand area) and background; where the objective sector is the ROI in white and the background sector in black.

\section{E. Algorithm of the proposed approach}

The hand vein recognition approach uses multiclassifier CNN, Alex Net, VGG16, VGG19, and the proposed approach. This approach was tested over a dataset after the Hand Vein Pattern Post-processing stage. As shown in Fig. (5). the selection of an optimum threshold to become the maximum system accuracy by analyzing the approach over the complete dataset is illustrated. To assess the vein patterns, multi metrics such as accuracy, FAR, FRR, precision, recall, and F.score were utilized.

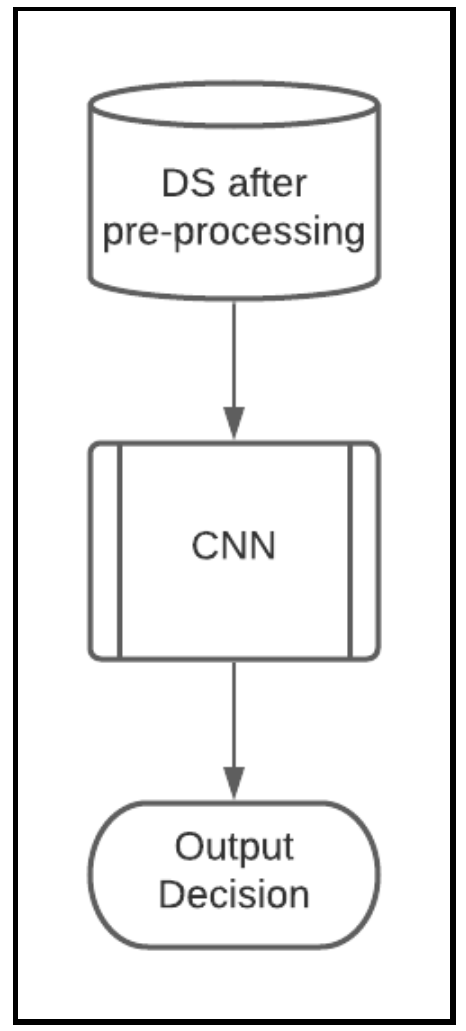

Fig. (5): Flowchart of the proposed approach

In the proposed model, four convolution layers which are followed by different filters of size $3 * 3$ for the first and second layers and $5 * 5$ for third and fourth layers with stride(2). Then an only max-pooling layer applied afterward using 3 fully linked layer with Relu and softmax layer with a single dropout layer 0.4 as displayed in 
Table (1) :Algorithm of the planned approach

\section{Algorithm (1 ): proposed model}

Input : Hand vein images

Output : Hand vein classifier

\section{Initialization :}

1 : Import numpy, pandas, sklearn, matplotlib, keras and tensor flow libraries

2 Set Batch_size,image_width,image_high,epoch

Train_datagen=keras.preprocessing.image.ImageDataGenerator( rescale $=1 . / 255$,

shear_range $=0.2$,

zoom_range $=0.2$,

horizontal_flip=True)

3

: $\quad$ Test_datagen=keras.preprocessing.image.ImageDataGenerator $($ rescale $=1 . / 255)$

4

Training_set $=$ Train_datagen.flow_from_directory (

'training_folder',

target_size $=($ image_width,image_high $)$,

batch_size $=$ Batch_size,

class_mode='categorical')

5

test_set $=$ test_datagen.flow_from_directory (

'test_folder',

: target_size=( image_width,image_high $)$,

batch_size $=$ Batch_size,

class_mode='categorical')

$6 \quad$ : Training_Model

7

Training_Model.compile(loss='categorical_crossentropy',optimizer=

$\operatorname{Adam}(\operatorname{lr}=0.0001)$, metrics $=[$ 'categorical_accuracy'] $)$

8

Training_Model.fit_generator(training_set, steps_per_epoch= number of training_set/batch_size, epochs=epoch, validation_data=test_set,

validation_steps= number of test_set/batch_size) 
Table (2): The construction of the vgg16 model

\begin{tabular}{|c|c|c|}
\hline Layer (type) & Output Shape & Param \# \\
\hline conv2d (Conv2D) & (None, $55,55,96$ ) & 34944 \\
\hline batch normalization & (Batc (None, $55,55,96$ ) & 384 \\
\hline max_pooling2d & (MaxPooling (None, 27, 27, 96) & 0 \\
\hline conv2d (Conv2D) & (None, $23,23,256$ ) & 614656 \\
\hline batch_normalization & (Batc (None, 23, 23, 256) & 1024 \\
\hline max_pooling2d & (MaxPooling (None, 11, 11, 256) & 0 \\
\hline conv2d (Conv2D) & (None, 9, 9, 384) & 885120 \\
\hline batch_normalization & (Batc (None, 9, 9, 384) & 1536 \\
\hline conv2d(Conv2D) & (None, $7,7,384$ ) & 1327488 \\
\hline batch_normalization & (Batc (None, 7, 7, 384) & 1536 \\
\hline conv2d (Conv2D) & (None, 5, 5, 256) & 884992 \\
\hline batch_normalization & (Batc (None, $5,5,256)$ & 1024 \\
\hline max_pooling2d & (MaxPooling (None, 2, 2, 256) & 0 \\
\hline flatten (Flatten) & (None, 1024) & 0 \\
\hline dense (Dense) & (None, 4096) & 4198400 \\
\hline dropout (Dropout) & (None, 4096) & 0 \\
\hline dense (Dense) & (None, 4096) & 16781312 \\
\hline dropout (Dropout) & (None, 4096) & 0 \\
\hline dense (Dense) & (None, 10) & 40970 \\
\hline
\end{tabular}


Table (3): The construction of the vgg19 model

\begin{tabular}{|c|c|c|}
\hline Layer (type) & Output Shape & Param \# \\
\hline conv2d (Conv2D) & (None, 227, 227, 64) & 1792 \\
\hline conv2d (Conv2D) & (None, 227, 227, 64) & 36928 \\
\hline max_pooling2d & (None, $113,113,64)$ & 0 \\
\hline conv2d (Conv2D) & (None, $113,113,128$ ) & 73856 \\
\hline conv2d (Conv2D) & (None, $113,113,128$ ) & 147584 \\
\hline max_pooling2d & (None, 28, 28, 256) & 0 \\
\hline conv2d (Conv2D) & (None, 28, 28, 256) & 295168 \\
\hline conv2d (Conv2D) & (None, 28, 28, 256) & 590080 \\
\hline conv2d(Conv2D) & (None, 28, 28, 256) & 590080 \\
\hline conv2d (Conv2D) & (None, 28, 28, 256) & 590080 \\
\hline max_pooling2d & (None, 28, 28, 512) & 0 \\
\hline conv2d (Conv2D) & (None, 28, 28, 512) & 1180160 \\
\hline conv2d (Conv2D) & (None, 28, 28, 512) & 2359808 \\
\hline conv2d (Conv2D) & (None, 28, 28, 512) & 2359808 \\
\hline conv2d (Conv2D) & (None, 28, 28, 512) & 2359808 \\
\hline max_pooling2d & (None, $14,14,512$ ) & 0 \\
\hline conv2d (Conv2D) & (None, $14,14,512$ ) & 2359808 \\
\hline conv2d (Conv2D) & (None, $14,14,512$ ) & 2359808 \\
\hline conv2d (Conv2D) & (None, 14, 14, 512) & 2359808 \\
\hline conv2d (Conv2D) & (None, $14,14,512$ ) & 2359808 \\
\hline max_pooling2d & (None, $7,7,512$ ) & 0 \\
\hline flatten (Flatten) & (None, 25088) & 0 \\
\hline dense (Dense) & (None, 4096) & 102764544 \\
\hline dropout (Dropout) & (None, 4096) & 0 \\
\hline dense (Dense) & (None, 4096) & 16781312 \\
\hline dropout (Dropout) & (None, 4096) & 0 \\
\hline dense (Dense) & (None, 20) & 81940 \\
\hline
\end{tabular}


Table (4): The construction of training cnn for the proposed model

\begin{tabular}{|c|c|c|}
\hline Layer (type) & Output Shape & Param \# \\
\hline conv2d (Conv2D) & (None, 225, 225, 32) & 896 \\
\hline batch normalization & (None, 225, 225, 32) & 128 \\
\hline max_pooling2d & (None, $112,112,32$ ) & 0 \\
\hline dropout (Dropout) & (None, $112,112,32$ ) & 0 \\
\hline conv2d (Conv2D) & (None, $110,110,32$ ) & 9248 \\
\hline batch_normalization & (None, 110, 110, 32) & 128 \\
\hline max_pooling2d & (None, $55,55,32$ ) & 0 \\
\hline dropout (Dropout) & (None, $55,55,32$ ) & 0 \\
\hline conv2d (Conv2D) & (None, 51, 51, 64) & 51264 \\
\hline batch_normalization & (None, 51, 51, 64) & 256 \\
\hline max_pooling2d & (None, 25, 25, 64) & 0 \\
\hline dropout (Dropout) & (None, 25, 25, 64) & 0 \\
\hline conv2d (Conv2D) & (None, 21, 21, 64) & 102464 \\
\hline batch_normalization & (None, 21, 21, 64) & 256 \\
\hline max_pooling2d & (None, 10, 10, 64) & 0 \\
\hline dropout (Dropout) & (None, 10, 10, 64) & 0 \\
\hline flatten (Flatten) & (None, 6400) & 0 \\
\hline dense (Dense) & (None, 512) & 3277312 \\
\hline dense (Dense) & (None, 128) & 65664 \\
\hline tuhdropout (Dropout) & (None, 128) & 0 \\
\hline dense (Dense) & (None, 10) & 1290 \\
\hline
\end{tabular}




\section{Experimental Result}

The proposed approaches are applied employing a laptop background (Intel(R) Core (MT) i7-7700 HQ CPU @ $2.80 \mathrm{GHz}$, win10 64-bit operating approach, RAM 16 GB, 256 GB SSD, NVIDIA GTX 1060 graphics card, and implemented in python 3.6 contains a deep learning library.
The assessment metrics engaged here are accurate, FAR, FRR, precision, recall, and F.score. The performance characteristics of the multi-classifier in standings of Accuracy are schemed in Fig. (6) for Alexnet, in Fig. (7) for VGG16, in Fig. (8) for VGG19, and in Fig. (9) for the proposed model.

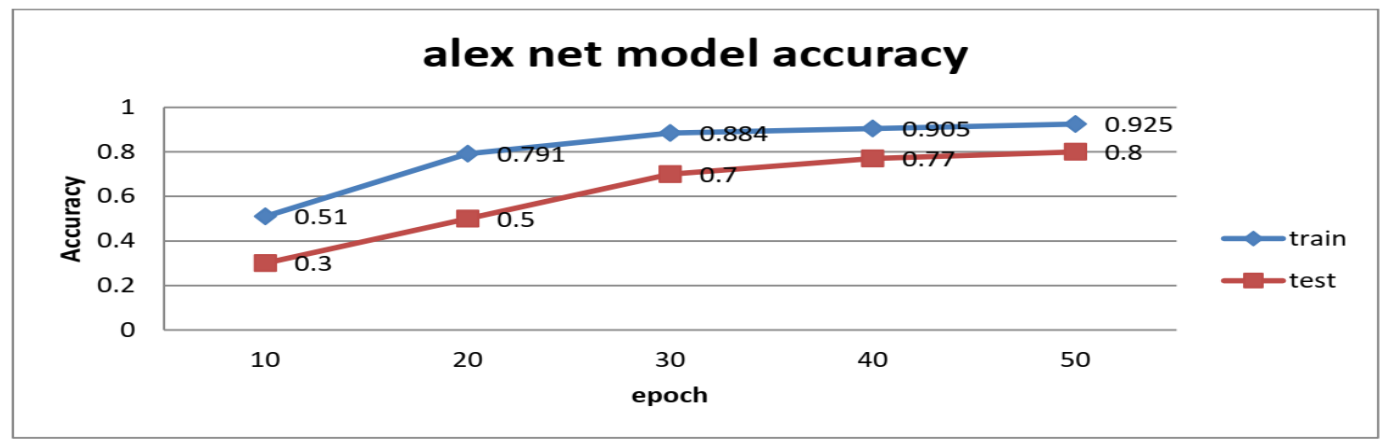

Fig. (6): Accuracy analysis of Alexnet

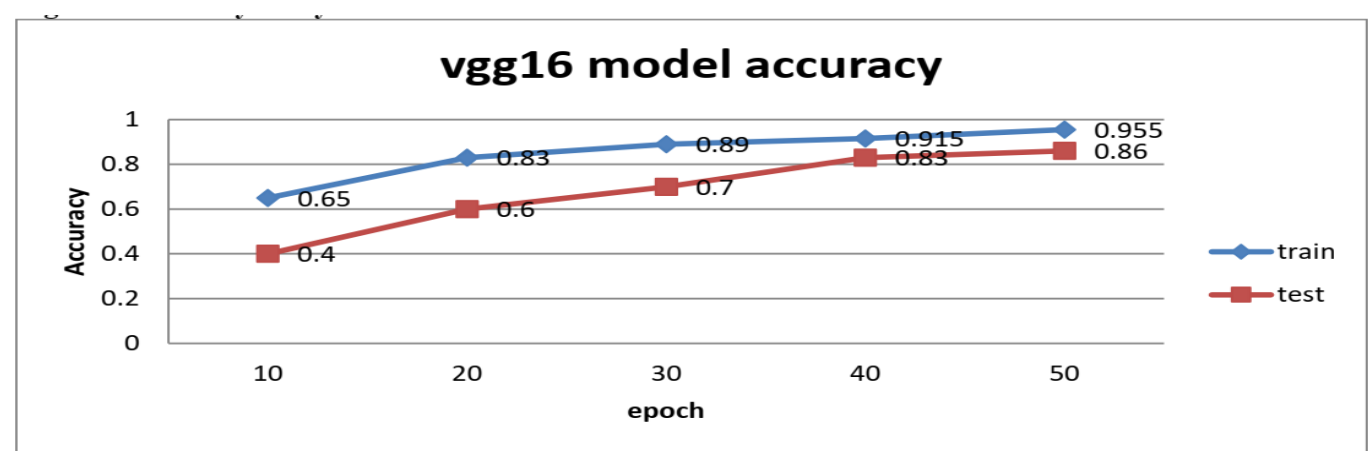

Fig. (7): Accuracy analysis of VGG16

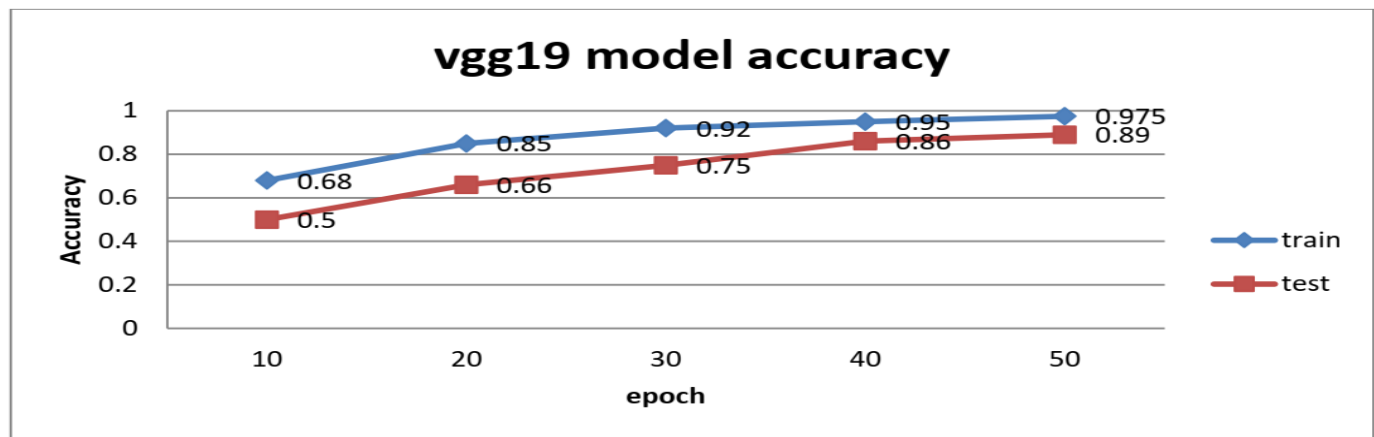

Fig. (8): Accuracy analysis of VGG19

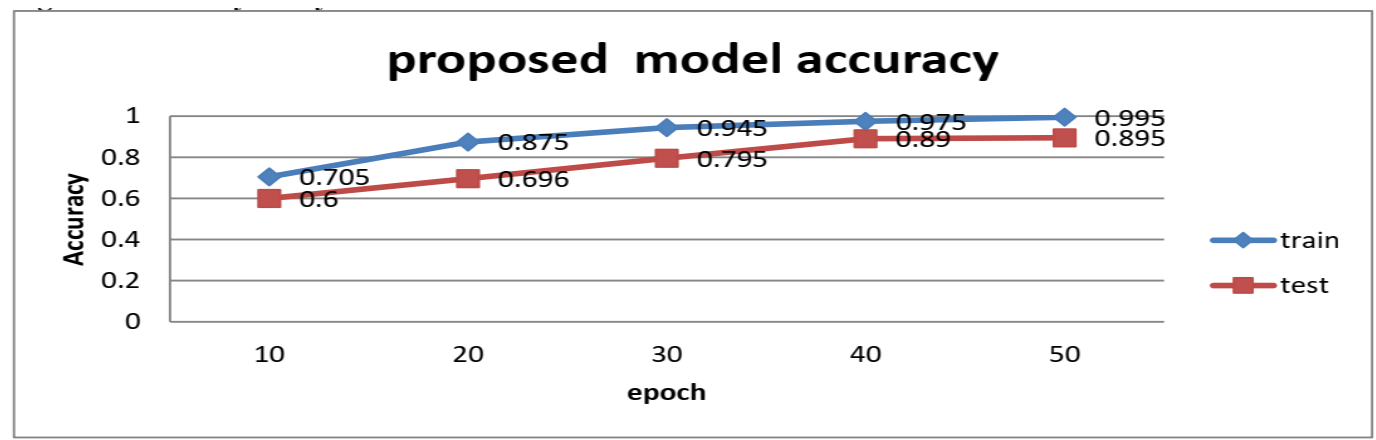

Fig. (9): Proposed model accuracy analysis 
At this point, for poorer values of threshold FAR are from top to bottom, and as threshold growth rates FAR reductions. Likewise, for minor values of threshold FRR,they are low and as the threshold rises, FRR also rises the concert features of the multiclassifier in standings of FRR ( Fig. (10) for FAR (Fig. (11) for the four classifiers.

A considerably improved method to assess the concert of a classifier is the appearance at the confusion matrix. The confusion matrix provides a percentage of statistics. The appearance of the accuracy of the PT is significantly unique for the classifier and is used sideways with another metric called recall (sensitivity). After that it is expedient to associate precision and recall hooked on a particular metric named the F1score (the harmonic mean) as illustrated in Fig. (12).

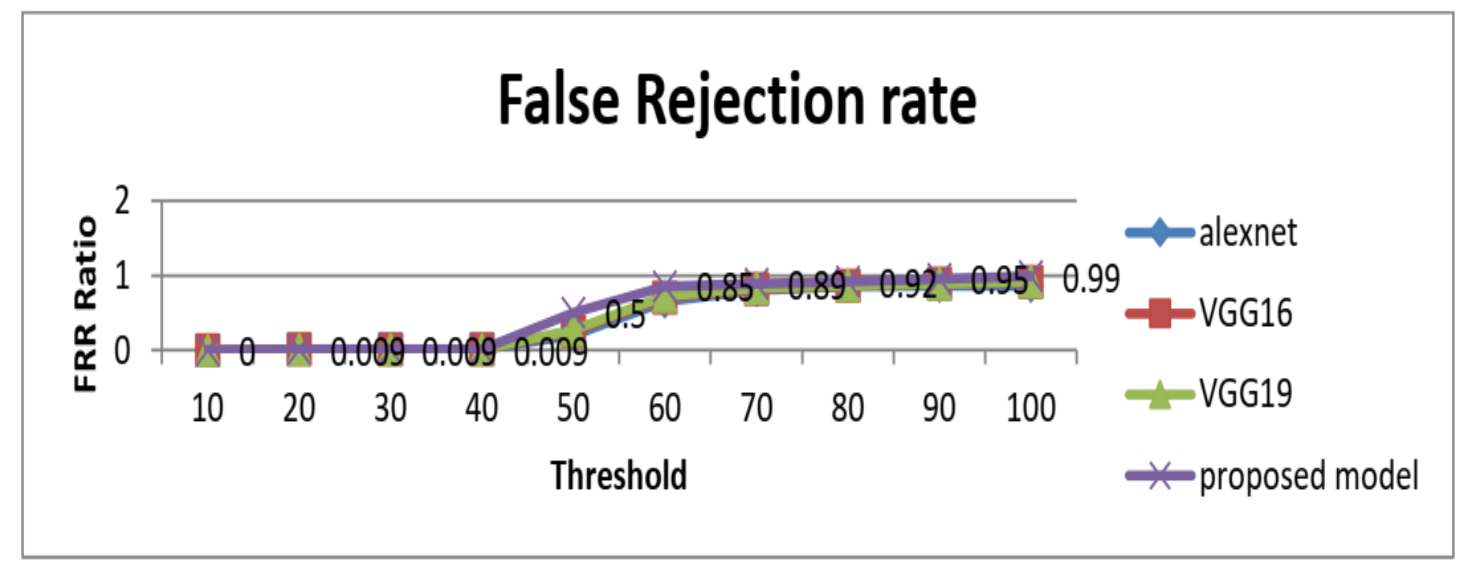

Fig. (10): Plot of FRR for Alexnet, VGG16, VGG19, and proposed model

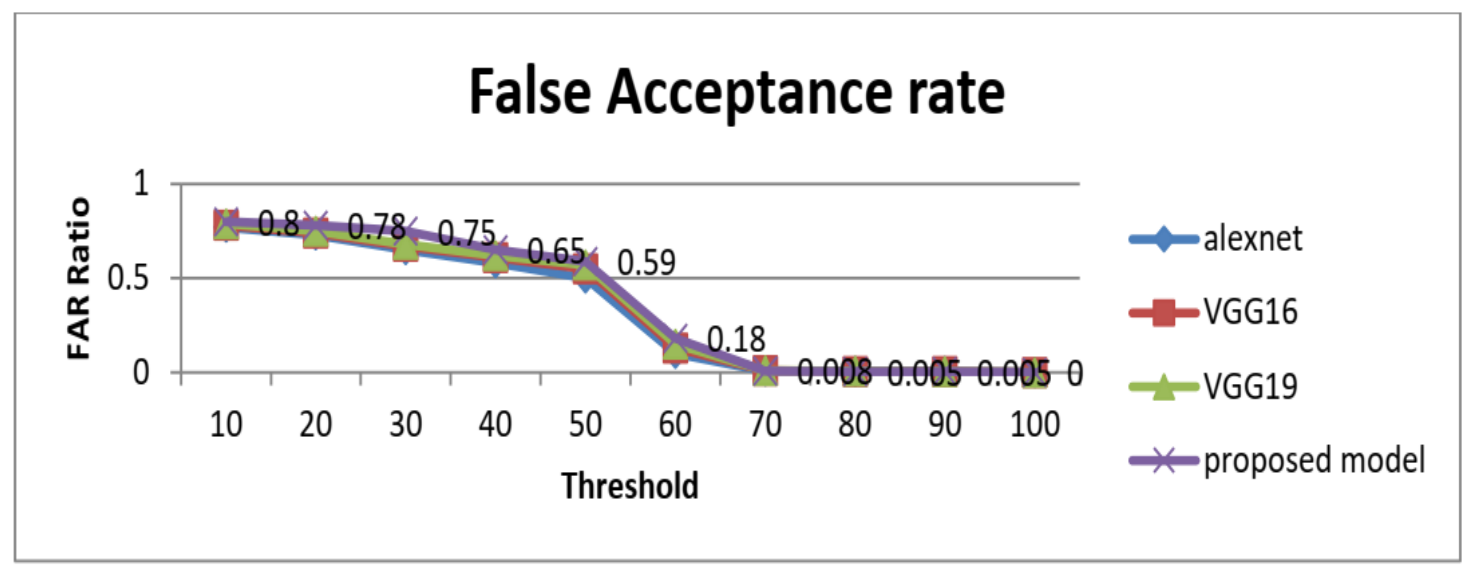

Fig. (11): Plot of FAR for Alexnet, VGG16, VGG19, and proposed model

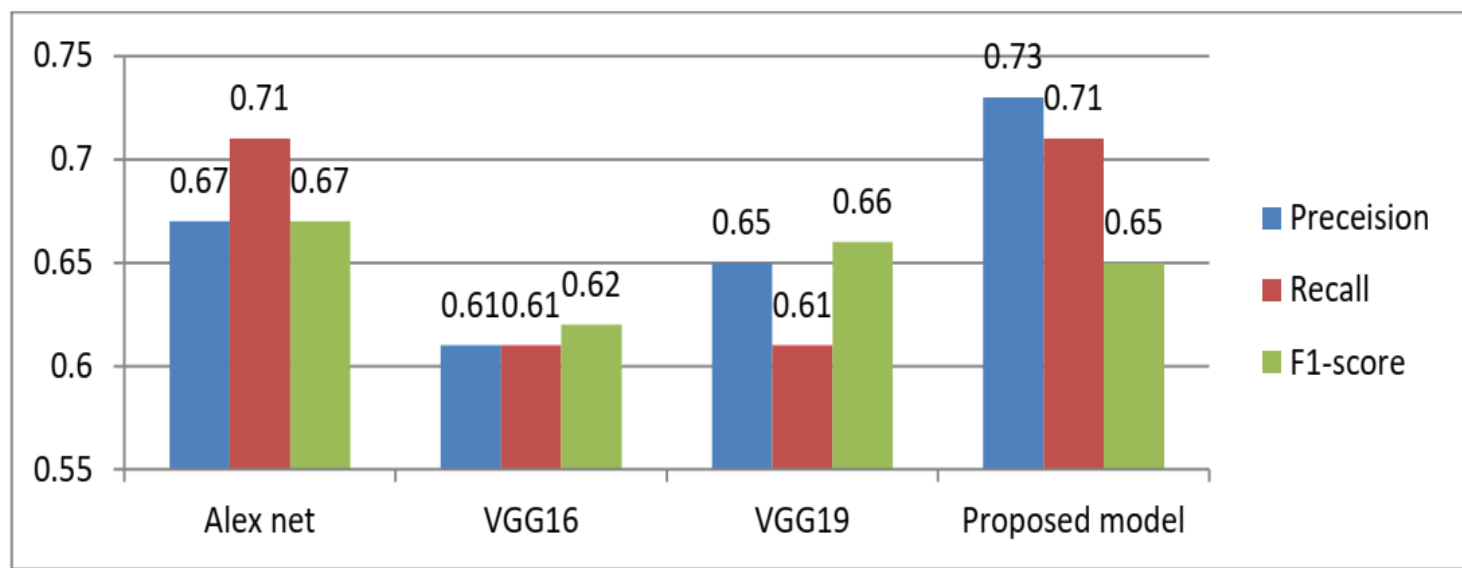

Fig. (12): Avg. Of precision, recall, and f-score for 100 objects 
Table (5): Comparison of the proposed approach and vein recognition methods

\begin{tabular}{|c|c|c|c|c|c|}
\hline number of references & proposed technique & number of objects & FAR & FRR & accuracy \\
\hline \{zhong, $2019 \# 155\}$ & $\begin{array}{l}\text { the deep hashing network } \\
\text { (dhn) }\end{array}$ & $\begin{array}{l}2040 \text { (50 male, } 52 \\
\text { female) }\end{array}$ & $0.74 \%$ & $5.75 \%$ & $95 \%$ \\
\hline$\{$ kauba, $2019 \# 156\}$ & capturing device & 840 & 0.329 & 86.12 & $93 \%$ \\
\hline \{chuang, $2018 \# 157\}$ & dynamic pattern tree (dpt) & 308 & 0.488 & 6.89 & $95 \%$ \\
\hline$\{$ wan, $2017 \# 159\}$ & different depth cn & 102 & - & . & $99.7 \%$ \\
\hline \{al-johani, 2020 \#160\} & transfer learning & 100 & - & - & 99.33 \\
\hline$\{$ dallas, $2020 \# 158\}$ & CNN and devops & 502 & - & - & $98.34 \%$ \\
\hline$\{$ Hong, 2017 \#223\} & CNN(VGG-16) & $\begin{array}{l}20 \text { persons with } 2 \\
\text { finger-vein }\end{array}$ & - & 0.97 & - \\
\hline$\{$ Cherrat, 2020 \#224\} & $\mathrm{CNN}$ & 106 finger vein & - & - & $99.13 \%$ \\
\hline proposed approach & $\begin{array}{l}\text { average filter } 9 * 9 \text { - relu } \\
\text { activation function with } \\
32,32,64,64\end{array}$ & 100 & 0.8 & 0.99 & $99.9 \%$ \\
\hline
\end{tabular}

Investigation outcomes demonstrate that this algorithm has accomplished, decent recognition result on the identified datasets; on the other hand, it is rare to discuss which one of the algorithms can be useful to other datasets. So, it will be of excessive consequence to suggest an operational hand vein recognition approach for widespread for multiple datasets.

\section{V.Conclusion}

Identification subject to hand-vein forms seems as an advantageous application where the attractiveness of vein patterns in the hand-vein area is requested to be unique to an object even for identical twins. This study proposes an approach for pre-processing for poor precise capturing of dataset images. Then the data augmentation for more varieties was applied. This study has applied hand-vein recognition by using deep learning. The proposed approach can be used for noisy dataset with the vascular tube. The pre-trained CNN model (VGG-16, VGG-19,ALEX-net and the proposed approach )established in this study is made following the techniques by other investigators for reasonable performance assessment. In total, 1575 hand vein images from 100 subject is used to authenticate the suggested hand vein recognition approach. A noticeable accuracy $(>99.8 \%)$ and FRR $(0.99 \%)$ was obtained.

In conclusion, using the proposed approach gives the most highly accurate rate for feature extraction. Finally, this effort would be a helpful opening point for new approaches, and a mutual ground for a widespread range of benefits in the space of other biometrics and handvein identification. The comparison confirms that the Deep learning models are the optimum ones based on the interval.

\section{REFERENCES}

1. AlMahafzah, H. and M.Z. AlRwashdeh, A Survey of Multibiometric Systems. ArXiv, 2012. abs/1210.0829.

2. Delac, K. and M. Grgic, A survey of biometric recognition methods. 2004. 184-193.

3. Qin, H. and M.A. El-Yacoubi, Deep Representation-Based Feature Extraction and Recovering for Finger-Vein Verification. IEEE Transactions on Information Forensics and Security, 2017. 12(8): p. 1816-1829.

4. Verma, D. and S. Dubey, Fuzzy least brain storm optimization and entropy-based Euclidean distance for multimodal vein-based recognition system. Journal of Central South University, 2017. 24(10): p. 2360-2371.

5. Wan, H., et al., Dorsal hand vein recognition based on convolutional neural networks. 2017 IEEE International Conference on Bioinformatics and Biomedicine (BIBM), 2017: p. 1215-1221.

6. Kim, W., Vein Enhancement Using a Dark Diffusion Prior. IEEE Signal Processing Letters, 2018. 25(9): p. 1325-1329. 
7. Bharathi, S. and R. Sudhakar, Biometric recognition using finger and palm vein images. Soft Computing, 2019. 23(6): p. 1843-1855.

8. Deokar, S. and S. Talele. Literature Survey of Biometric Recognition Systems. 2014.

9. Qin, H., et al., An Iterative Deep Neural Network for Hand-Vein Verification. IEEE Access, 2019. PP: p. 1-1.

10. Bashar, K. and M. Murshed. Texture Based Vein Biometrics for Human Identification: A Comparative Study. in 2018 IEEE 42nd Annual Computer Software and Applications Conference (COMPSAC). 2018.

11. Zhong, D., et al. Palmprint and Dorsal Hand Vein Dualmodal Biometrics. in 2018 IEEE International Conference on Multimedia \& Expo Workshops (ICMEW). 2018.

12. Ahmed, M.A. and A.-B.M. Salem, ANALYSIS OF HAND VEINS AND MULTIMODAL PATTERN RECOGNITION ALGORITHMS AND SYSTEMS.

13. Chantaf, S., A. Hilal, and R. Elsaleh, Palm Vein Biometric Authentication Using Convolutional Neural Networks. 2020. p. 352-363.

14. Estacio, R.R. and N.B. Linsangan. A Rotation Invariant Algorithm for Bimodal Hand Vein Recognition System. in 2018 IEEE 10th International Conference on Humanoid, Nanotechnology, Information Technology,Communication and Control, Environment and Management (HNICEM). 2018.

15. Bhagat, K.S., et al. Iris recognition using radon transform and GLCM. in 2017 International Conference on Advances in Computing, Communications and Informatics (ICACCI). 2017.

16. Lee, J.-C., T.-M. Lo, and C.-P. Chang, Dorsal hand vein recognition based on directional filter bank. Signal, Image and Video Processing, 2016. 10(1): p. $145-152$.

17. Trabelsi, R., D. Alima, and D. Sellami, Hand vein recognition system with circular difference and statistical directional patterns based on an artificial neural network. Multimedia Tools and Applications, 2016. 75: p. 687-707.

18. Yin, Y., L. Liu, and X. Sun. SDUMLA-HMT: A Multimodal Biometric Database. in CCBR. 2011.

19. Wang, L., G. Leedham, and S.-Y. Cho, Minutiae feature analysis for infrared hand vein pattern biometrics. Pattern Recognit., 2008. 41: p. 920-929.

20. Li, J., et al. An Approach for Biometric Verification Based on Human Body Communication using
Convolutional Neural Network. in 2019 IEEE 9th International Conference on Consumer Electronics (ICCE-Berlin). 2019.

21. Chuang, S.-J., Vein recognition based on minutiae features in the dorsal venous network of the hand. Signal, Image and Video Processing, 2018. 12(3): p. 573-581.

22. Wan, H., et al., Dorsal hand vein recognition based on convolutional neural networks. 2017. 12151221.

23. Kim, W., Vein Enhancement Using a Dark Diffusion Prior. IEEE Signal Processing Letters, 2018. 25: p. 1325-1329.

24. Song, J., W. Kim, and K. Park, Finger-Vein Recognition Based on Deep DenseNet Using Composite Image. IEEE Access, 2019. PP: p. 1-1.

25. Zhong, D., H. Shao, and S. Liu, Towards application of dorsal hand vein recognition under uncontrolled environment based on biometric graph matching. IET Biometrics, 2019. 8(2): p. 159-167.

26. Thakuria, H., et al. A comparative study of vein pattern recognition for biometrie authentication. in 2017 8th IEEE Annual Information Technology, Electronics and Mobile Communication Conference (IEMCON). 2017.

27. Diab, A.A.Z., et al., Fuel Cell Parameters Estimation via Marine Predators and Political Optimizers. IEEE Access, 2020. 8: p. 166998167018.

28. Song, J.M., W. Kim, and K.R. Park, Finger-Vein Recognition Based on Deep DenseNet Using Composite Image. IEEE Access, 2019. 7: p. 6684566863.

29. Zhong, D., H. Shao, and X. Du, A Hand-Based Multi-Biometrics via Deep Hashing Network and Biometric Graph Matching. IEEE Transactions on Information Forensics and Security, 2019. 14(12): p. 3140-3150.

30. Yüksel, A., L. Akarun, and B. Sankur. Biometric Identification through Hand Vein Patterns. in 2010 International Workshop on Emerging Techniques and Challenges for Hand-Based Biometrics. 2010.

31. Jia, X., F. Sun, and D. Chen, Vein Recognition Algorithm Based on Transfer Nonnegative Matrix Factorization. IEEE Access, 2020. 8: p. 101607101615.

32. Yuhang, D., Z. Dayan, and W. Kejun. A study of hand vein recognition method. in IEEE International Conference Mechatronics and Automation, 2005. 2005.

Arab J. Nucl. Sci. Appl., Vol. 54, 4, (2021) 
33. Kumar, A. and K.V. Prathyusha, Personal Authentication Using Hand Vein Triangulation and Knuckle Shape. IEEE Transactions on Image Processing, 2009. 18(9): p. 2127-2136.

34. Kumar, R., R.C. Singh, and S. Kant. Dorsal Hand Vein-Biometric Recognition Using Convolution Neural Network. in International Conference on Innovative Computing and Communications. 2021. Singapore: Springer Singapore.

35. Pflug, A., D. Hartung, and C. Busch, Feature extraction from vein images using spatial information and chain codes. Information Security Technical Report, 2012. 17: p. 26-35.

36. Huang, D., et al., Hand-Dorsa Vein Recognition by Matching Local Features of Multisource Keypoints. IEEE Transactions on Cybernetics, 2015. 45(9): p. 1823-1837.

37. Bhilare, S., et al., Single-sensor hand-vein multimodal biometric recognition using multiscale deep pyramidal approach. Machine Vision and Applications, 2018. 29.

38. Cross, J.M. and C.L. Smith. Thermographic imaging of the subcutaneous vascular network of the back of the hand for biometric identification. in Proceedings The Institute of Electrical and Electronics Engineers. 29th Annual 1995 International Carnahan Conference on Security Technology. 1995.

39. Im, S.K., et al., An biometric identification system by extracting hand vein patterns. Journal of the Korean Physical Society, 2001. 38: p. 268-272.

40. Bhattacharyya, D., et al. Biometric Authentication Using Infrared Imaging of Hand Vein Patterns. in Information Security and Assurance. 2010. Berlin, Heidelberg: Springer Berlin Heidelberg.

41. Miura, N., A. Nagasaka, and T. Miyatake, Feature extraction of finger-vein patterns based on repeated line tracking and its application to personal identification. Machine Vision and Applications, 2004. 15(4): p. 194-203.

42. Wang, L. and G. Leedham, Near-and Far-Infrared Imaging for Vein Pattern Biometrics. 2006. 52-52.
43. Lajevardi, S.M., et al., Hand vein authentication using biometric graph matching. IET Biometrics, 2014. 3(4): p. 302-313.

44. Sontakke, B., V. Humbe, and P. Yannawar, Dorsal Hand Vein Authentication System: A Review. Journal of Scientific Research and Development, 2017. 6: p. 511-514.

45. Raghavendra, R. and C. Busch, Hand Dorsal Vein Recognition: Sensor, Algorithms and Evaluation. 2015.

46. Rossan, I. and M. Heenaye- Mamode Khan, Impact of Changing Parameters when Preprocessing Dorsal Hand Vein Pattern. Procedia Computer Science, 2014. 32: p. 513-520.

47. Qin, H. and M. El Yacoubi, Deep RepresentationBased Feature Extraction and Recovering for Finger-Vein Verification. IEEE Transactions on Information Forensics and Security, 2017. PP: p. 11.

48. Kang, W. and Q. Wu, Contactless Palm Vein Recognition Using a Mutual Foreground-Based Local Binary Pattern. IEEE Transactions on Information Forensics and Security, 2014. 9(11): p. 1974-1985.

49. Zhou, Y., et al., Palm-vein classification based on principal orientation features. PLoS One, 2014. 9(11): p. e112429.

50. Reşit Kavsaoğlu, A., K. Polat, and M. Recep Bozkurt, A novel feature ranking algorithm for biometric recognition with PPG signals. Comput Biol Med, 2014. 49: p. 1-14.

51. Prabu, S., M. Lakshmanan, and V.N. Mohammed, A Multimodal Authentication for Biometric Recognition System using Intelligent Hybrid Fusion Techniques. J Med Syst, 2019. 43(8): p. 249.

52. Sohrabi Safa, N., A. Norjihan, and M.A. Ismail, An artificial neural network classification approach for improving accuracy of customer identification in ecommerce. Malaysian Journal of Computer Science, 2014. 27: p. 171-185.

53. Lecun, Y., et al., Gradient-Based Learning Applied to Document Recognition. Proceedings of the IEEE, 1998. 86: p. 2278-2324. 